\title{
A rare cause of lower abdominal and pelvic mass, primary tuberculous psoas abscess: a case report Ana Paula Vaz*1, Joana Gomes ${ }^{2}$, Joana Esteves ${ }^{3}$, Aurora Carvalho ${ }^{2,4}$ and Raquel Duarte $2,4,5$
}

Address: ${ }^{1}$ Department of Pulmonology, Hospital Sao Joao, Alameda Professor Hernani Monteiro 4300-319, Oporto, Portugal, ${ }^{2}$ Department of Pulmonology, Centro Hospitalar de Vila Nova de Gaia/Espinho, Rua Conceicao Fernandes, 4434-502, Vila Nova de Gaia, Portugal, ${ }^{3}$ Department of General Surgery, Centro Hospitalar de Vila Nova de Gaia/Espinho, Rua Conceicao Fernandes, 4434-502, Vila Nova de Gaia, Portugal, ${ }^{4}$ Centro de Diagnostico Pneumologico de Vila Nova de Gaia, Rua Conselheiro Veloso da Cruz, 4400-092, Vila Nova de Gaia, Portugal and ${ }^{5}$ Oporto Medical Faculty, Oporto University, Alameda Professor Hernani Monteiro 4300-319, Oporto, Portugal

Email: Ana Paula Vaz* - vaz.anapaula@gmail.com; Joana Gomes - jolobogomes@hotmail.com; Joana Esteves - joanasalles@gmail.com; Aurora Carvalho - dra.aurora.carvalho@gmail.com; Raquel Duarte - raquelafduarte@gmail.com

* Corresponding author

\section{Published: 3 November 2009}

Cases Journal 2009, 2:182 doi:10.1186/1757-1626-2-182

This article is available from: http://www.casesjournal.com/content/2/I/l82

(C) 2009 Vaz et al; licensee BioMed Central Ltd.

This is an Open Access article distributed under the terms of the Creative Commons Attribution License (http://creativecommons.org/licenses/by/2.0), which permits unrestricted use, distribution, and reproduction in any medium, provided the original work is properly cited.
Received: 19 October 2009
Accepted: 3 November 2009

\begin{abstract}
Introduction: Tuberculous psoas abscess was usually associated to complicate Pott's disease, but it can also be secondary to direct extension from other adjacent structures or haematogenous spread from an occult source. However, the occurrence of this entity as the presenting manifestation of tuberculosis, without evidence of active infection elsewhere, has been seldom reported.

Case Presentation: We report a clinical case of a 64-year-old immunocompetent female that presented with left lower abdominal pain and a soft tissue mass over the left iliac fossa and inguinal regions due to a primary tuberculous psoas abscess. Early diagnosis and prompt treatment with percutaneous drainage guided by ultrasound along with antituberculous drugs, lead to a satisfactory outcome.

Conclusion: The purpose of this case report is to point out attention to the diagnostic challenge of tuberculous psoas abscess in the absence of tuberculosis in other organs or a predisposing condition. A brief review of the literature about its epidemiology, etiology, clinical features and management is discussed over the text.
\end{abstract}

\section{Introduction}

Psoas abscess is regarded as a rare disease in the medical literature. In 1992, the worldwide reported occurrence of psoas abscess was 12 cases per year [1]. This was a significant increase from the calculated occurrence of 3.9 cases per year before 1985. Nowadays, the truly incidence is unknown but it has probably increased due to improvements in diagnostic techniques $[2,3]$.
The causes of psoas abscess have also changed in the last decades. At the beginning of the 20th century, psoas abscess was mainly caused by tuberculosis (TB) of the spine (Pott's disease). With the decline of Mycobacterium tuberculosis (MT) as a major pathogen in developed countries, psoas abscess was mostly seen secondary to diseases of the digestive tract $[2,4]$. In recent years, a primary psoas abscess due to haematogenous spread from an 
occult source is more common, especially in immunocompromised and older patients $[2,4]$. In addition, TB is increasing again in some risk groups.

The psoas abscess may be clinically difficult to diagnose because of its rarity, insidious onset of the disease and nonspecific clinical presentation, which can cause diagnostic delays, resulting in high morbidity. Therefore, early diagnosis and appropriate management remain a challenge for clinicians.

We report a case of a primary psoas abscess due to MT infection in an immunocompetent female that presented with left lower abdominal pain and a soft tissue mass over the left iliac fossa (LIF) and inguinal regions.

\section{Case Presentation}

A 64-year-old Portuguese white female was referred to the emergency department with progressive left lower quadrant abdominal pain radiating to the ipsilateral hip and thigh, over the previous 2 months. Palpable soft tissue mass over the LIF and inguinal areas was noted 1 week before admission. Her medical history was negative, except for high blood pressure. There was no history of trauma, cutaneous or other infection. The patient denied fever, back pain, gastrointestinal or genitourinary symptoms, weight loss or weakness.

On admission, her general condition was good and she was not febrile. The chest was clear to auscultation and no adenopathy was found. Examination of the LIF and inguinal regions showed a fluctuant, warm, mildly tender mass, measuring $10 \times 5 \mathrm{~cm}$. Flexion and external rotation of the ipsilateral hip was painful. The neurological examination was normal.

Blood test showed an increased C-reactive protein (1.48 $\mathrm{mg} / \mathrm{dl}$ ), without any others abnormalities. Urine analysis was normal.

Abdominal, pelvic and tight ultrasound (US) revealed the presence of a hypoechoic collection with many internal echoes (Figure. 1) and the computed tomography (CT) established the diagnosis of a loculated fluid-density mass, measuring $20 \mathrm{~cm}$ of length, beginning on the LIF, involving the psoas muscle and extending inferiorly through the inguinal region until the root of the tight, with discrete infiltration of the subcutaneous tissues.

The patient initiated broad spectrum intravenous antibiotherapy and percutaneous drainage (PCD) guided by US was performed, with aspiration of grossly purulent fluid. A catheter was placed for 6 days, obtaining $110 \mathrm{ml}$ of pus. Microscopical examination of the fluid showed many polymorphonuclear cells without acid-fast bacilli and cultures for bacteria or fungi were negative. The microbiological study of blood, sputum and urine was also negative. There was no clinical evidence of other organs' involvement, namely the lung, the spine, the hip or the gastrointestinal or genitourinary tracts, which was also confirmed by radiological and endoscopic studies. Serologic HIV testing was negative.

A clinical and radiological improvement was seen, with the control CT, showing a residual collection confined to the subcutaneous region.
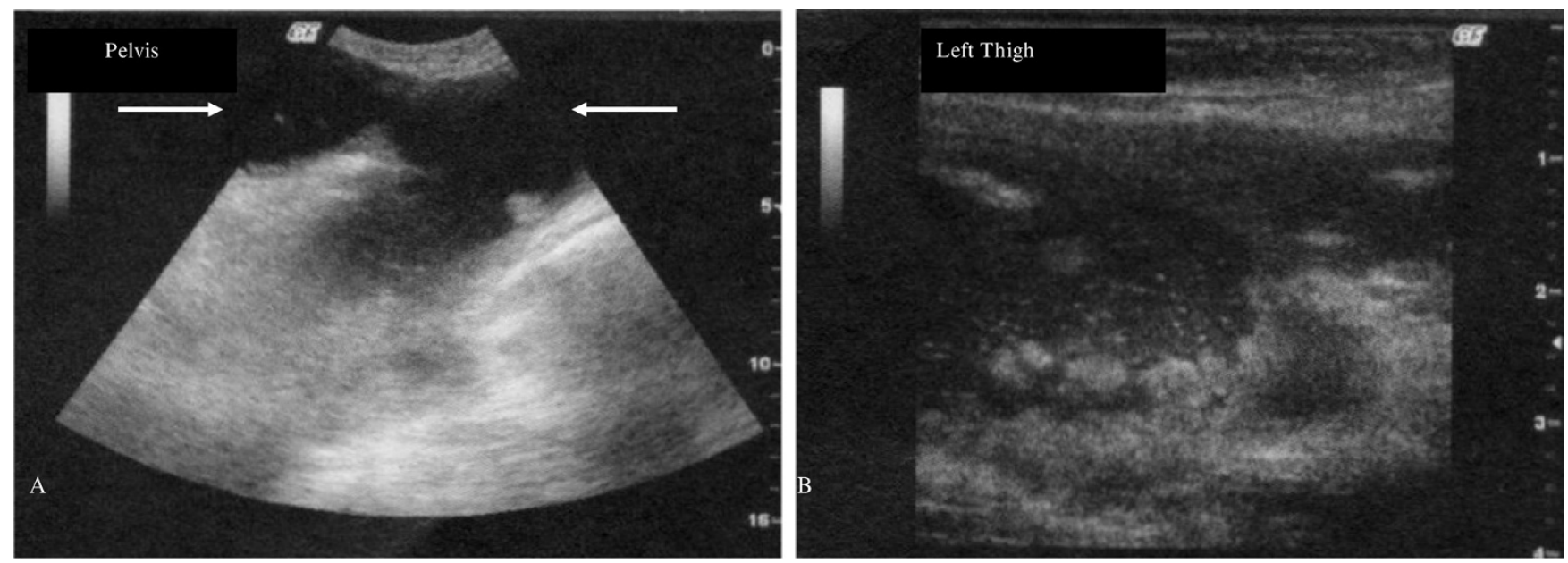

Figure I

Transverse abdominal, pelvic and left thigh ultrasound. Hypoechoic collection with many internal echoes beginning on the left iliac fossa, involving the psoas muscle (arrows) (A) and extending inferiorly through the inguinal region until the root of the tight, with discrete infiltration of the subcutaneous tissues (B). 
In the meanwhile, abscess culture was positive for $\mathrm{Myco-}$ bacterium tuberculosis complex and the patient was oriented to our unit and started directly observed therapy with 4 weight adjusted drugs, (daily isoniazid $300 \mathrm{mg}$, rifampicin $600 \mathrm{mg}$, pyrazinamide $1500 \mathrm{mg}$ and ethambutol $1200 \mathrm{mg}$ ). The specie was sensitive to all first line drugs in use. Two months later, the antituberculous treatment was reduced to 2 drugs, isoniazid and rifampicin. Almost after the 6 months of treatment, the patient is well and no signs of relapse have been detected.

\section{Discussion}

The iliopsoas compartment is an extraperitoneal space which contains the iliopsoas and iliacus muscles. The psoas major muscle arises from the transverse processes and bodies of the $12^{\text {th }}$ thoracic and all lumbar vertebrae. Superiorly it passes beneath the arcuate ligament of the diaphragm, proceeds downward across the brim of the lesser pelvis, passes beneath the inguinal ligament and in front of the capsule of the hip joint and ends in a tendon that receives nearly the whole of the fibres of the iliacus muscle and is inserted into the lesser trochanter of the femur. Thus, the space defined by the psoas fascia is a direct communication from the mediastinum to the thigh. It is innervated by the branches of L2, 3, and 4, and it is the primary flexor of the hip joint.

The psoas abscess may be classified as primary or secondary, depending on the presence or absence of underlying disease. Since this muscle lies in close proximity to organs such as the sigmoid colon, appendix, jejunum, ureters, abdominal aorta, kidneys, pancreas, spine, and iliac lymph nodes, any underlying disease in these organs can spread secondarily to the iliopsoas muscle [4]. In a review of 367 cases, Ricci et al noted that the most common cause of secondary psoas abscess was Crohn's disease (60\%) [5].

The etiology of primary psoas abscess is unclear, but lymphatic and hematogenous spread of an infectious process from an occult source in the body, often associated with immunosuppressant conditions like diabetes mellitus, renal failure, intravenous drug abuse, human immunodeficiency virus infection, malignancies and other chronic illness or trauma, has been implicated [4].

The review of Ricci et al noted differences in the aetiology of this entity. In secondary psoas abscess, cultures are often mixed with Escherichia coli and Bacteroides spp predominating [5]. On the other hand, Staphylococcus aureus is found in $88 \%$ of the cases of primary psoas abscess followed by streptococci (5\%) and Escherichia coli (3\%) [5]. In the literature, there are other reported causes of primary psoas abscess like brucellosis, trichinosis, typhilitis, pneumococcus or MT [6].
Iliopsoas abscess was first described by Mynter in 1881, who referred to this as psoitis; since then, iliopsoas abscess was characteristically a well recognised complication of TB of the spine [7]. Less frequently, tuberculous psoas abscess can be secondary to direct extension from other adjacent structures [8], or even haematogenous seeding from a distant site. However, the occurrence of tuberculous psoas abscess as the presenting manifestation of TB, without evidence of active infection elsewhere, has been seldom reported [6,9-12].

In the majority of these cases, as in our own case, those patients presented subacute or chronic symptoms and good general status. Although fever, abdominal or back pain and limitation of hip joint movements are the classical triad of psoas abscess, it can be presented only in 35\% of all patients [3]. Clinical suspicion, radiological study with US and CT, the last considered the gold standard, along with microbiological culture of the pus, are crucial to the diagnosis.

Besides that, ruling out another source of infection in the lung, spine, hip, genitourinary or gastrointestinal tracts should be also kept in mind. Since no tuberculous disease was found in other organs, this case was considered as a primary psoas abscess. The respiratory tract might have been the route of entry of bacilli and in an adult the reactivation of a quiescent tuberculous focus from an occult source may result in haematogenous spread of bacteria, even in the absence of a predisposing condition.

Treatment of tuberculous psoas abscess involved the use of appropriate antituberculous drugs along with drainage. PCD has been shown to be the first line treatment of abdominal abscess, including tuberculous, which renders surgery unnecessary in many cases [13]. Our patient underwent US guided PCD and is responding well to antituberculous therapy, which is in keeping with most of the patients reviewed in literature, which reports that primary psoas abscess seems to have a better prognosis than those secondary to other disease [5].

\section{Abbreviations}

CT: Computed tomography; LIF: Left iliac fossa; MT: Mycobacterium tuberculosis; PCD: Percutaneous drainage; TB: Tuberculosis; US: Ultrasound.

\section{Consent}

Written informed consent was obtained from the patient for publication of this case report and accompanying images. A copy of the written consent is available for review by the Editor-in-Chief of this journal.

\section{Competing interests}

The authors declare that they have no competing interests. 


\section{Authors' contributions}

APV, JG, JE, AC, RD analyzed and interpreted the patient data. They all gave a major contributor in writing the manuscript. All authors read and approved the final manuscript.

\section{References}

I. Gruenwald I, Abramson J, Cohen O: Psoas abscess: case report and review of the literature. J Urol 1992, 147:1624-1626.

2. Berge VM, Marie S, Kuipers T, Jansz AR, Bravenboer B: Psoas abscess: report of a series and review of the literature. Neth J Med 2005, 63(10):413-416.

3. Penado S, Espina B, Campo J: Absceso de psoas. Descripcion de una serie de 23 casos. Enferm Infecc Microbiol Clin 200I, 19:257-260.

4. Mallick I, Thoufeeq M, Rajendran T: Iliopsoas abscesses. Postgrad Med J 2004, 80:459-462.

5. Ricci MA, Rose FB, Meyer KK: Pyogenic psoas abscess: worldwide variations in etiology. World J Surg 1986, 10:834-843.

6. Cubukcu S, Gurbuz U, Çevilkol C, Aktan S, Tuncer T: Primary psoas abscess presented with only low back pain. Turk J Phys Med Rehab 2006, 52: I37-I 40.

7. Mynter H: Acute psoitis. Buffalo Med Surg J I88I, 21:202-210.

8. Chaoui F: Bilateral tuberculous abscess of the psoas without spinal involvement associated with colonic tuberculosis. Presse Med 1987, 16: I 100-1 I0I.

9. Younes M, Ayeche M, Bejia I, Hamida R, Dahmene J, Moula T: Abces tuberculeux du psoas sans atteinte rachidienne associee. $A$ propos d'un cas Rev Med Interne 2002, 23:549-553.

10. Mateos A, Monte R, Rodriguez A, Corredoira J: Primary Psoas abscess caused by Mycobacterium tuberculosis. Scand J Infect Dis 1998, 30(3):319.

II. Harrigan RA, Kauffman FH, Love MB: Tuberculous psoas abscess. J Emerg Med 1995, I3(4):493-498.

12. Chen YC, Chang SC, Hsieh WC, Luch KT, Shen MC: Non-spinal psoas abscess due to Mycobacterium tuberculosis in a patient with acquired immunodeficiency syndrome: a report case. J Formos Med Assoc 1994, 93(5):433-436.

13. Dinc H, Onder C, Turhan AU, Sari A, Aydin A, Yulug G, Gumele HR: Percutaneous catheter drainage of tuberculous and nontuberculous psoas abscesses. Eur J Radiol 1996, 23(2): I30-134.
Publish with Bio Med Central and every scientist can read your work free of charge

"BioMed Central will be the most significant development for disseminating the results of biomedical research in our lifetime. "

Sir Paul Nurse, Cancer Research UK

Your research papers will be:

- available free of charge to the entire biomedical community

- peer reviewed and published immediately upon acceptance

- cited in PubMed and archived on PubMed Central

- yours - you keep the copyright 